\title{
Effective behavioral intervention strategies using mobile health applications for chronic disease management: a systematic review
}

Jung-Ah Lee ${ }^{1}$, Mona Choi ${ }^{2^{*}}$, Sang A Lee ${ }^{3}$ and Natalie Jiang ${ }^{4}$

\begin{abstract}
Background: Mobile health (mHealth) has continuously been used as a method in behavioral research to improve self-management in patients with chronic diseases. However, the evidence of its effectiveness in chronic disease management in the adult population is still lacking. We conducted a systematic review to examine the effectiveness of mHealth interventions on process measures as well as health outcomes in randomized controlled trials (RCTs) to improve chronic disease management.
\end{abstract}

Methods: Relevant randomized controlled studies that were published between January 2005 and March 2016 were searched in six databases: PubMed, CINAHL, EMBASE, the Cochrane Library, PsycINFO, and Web of Science. The inclusion criteria were RCTs that conducted an intervention using mobile devices such as smartphones or tablets for adult patients with chronic diseases to examine disease management or health promotion.

Results: Of the $12 \mathrm{RCTs}$ reviewed, 10 of the mHealth interventions demonstrated statistically significant improvement in some health outcomes. The most common features of mHealth systems used in the reviewed RCTs were real-time or regular basis symptom assessments, pre-programed reminders, or feedbacks tailored specifically to the data provided by participants via mHealth devices. Most studies developed their own mHealth systems including mobile apps. Training of mHealth systems was provided to participants in person or through paper-based instructions. None of the studies reported the relationship between health outcomes and patient engagement levels on the mHealth system.

Conclusions: Findings from mHealth intervention studies for chronic disease management have shown promising aspects, particularly in improving self-management and some health outcomes.

Keywords: Mobile applications, Disease management, Mobile health, Chronic disease management, Self-management

\section{Background}

The prevalence of chronic diseases, such as cancer, cardiovascular diseases, chronic pain, diabetes, and respiratory diseases is continuously increasing with regard to an aging society worldwide. According to the World Health Organization, chronic diseases are the leading cause of mortality in the world, accounting for more than $60 \%$ of all deaths [1]. Chronic disease is, therefore, a global burden. For example, according to the report by

\footnotetext{
* Correspondence: monachoi@yuhs.ac

${ }^{2}$ College of Nursing, Mo-Im Kim Nursing Research Institute, Yonsei University, 50 Yonsei-ro, Seodaemun-gu, Seoul, Republic of Korea03722

Full list of author information is available at the end of the article
}

the Centers for Disease Control and Prevention (CDC) in the United States (US), about half of all American adults, approximately 117 million people, have one or more chronic disease conditions including heart disease, stroke, cancer, type 2 diabetes, obesity, or arthritis [2]. One in four adults in the US had two or more chronic diseases in 2012 [2]. Chronic diseases are the main cause of death among Americans, with 48\% dying from cancer or heart diseases in 2010 [3]. In 2010, about $86 \%$ of Americans' health care expenditure was for chronic disease treatment [4]. Therefore, chronic disease management is now a major public health issue in the US. Likewise, managing chronic diseases is also a challenge 
in other countries [5, 6]. For instance, over $40 \%$ of the population aged 15 years or older had a chronic disease condition in the European Union countries [5] and chronic diseases accounted for a substantial proportion of deaths throughout Southeast Asia [6].

With advances in mobile technologies, approaches based on mobile health (mHealth)-defined as "an area of electronic health (eHealth) with the provision of health services and information via mobile technologies such as mobile phones and Personal Digital Assistants (PDAs)" [7]-have been very popular in health care and public health [8-11]. Current evidence shows that the advantages of using mHealth devices are not only for the improvement of diagnosis and treatment but also the social connection with people [12]. Behavioral interventions using mobile applications (apps) on smartphones or tablet computers in enhancing self-management for patients with chronic diseases, such as heart failure [13] or diabetes [14], have been studied [9, 12]. For instance, a food intake diary, physical activity monitoring, and home blood sugar monitoring via mHealth systems are commonly used for diabetes management [14-16] while monitoring of weight, symptoms, and physical activity are common features of heart failure interventions $[13,17]$.

However, the evidence from current literature using the mHealth approach on improving health outcomes is inconsistent; some studies have shown that mHealthbased behavioral interventions are potentially effective in chronic disease management, whereas other studies did not obtain supportive results [9]. Previously, the evaluation of mHealth-based research focused on feasibility and acceptability of mHealth tools.

Rather than relying on feasibility research, which often does not utilize randomization in their intervention and/ or a control group, and often lacks an effective size, a number of systematic or integrated reviews examined randomized controlled trials (RCTs) in diabetes management. These studies have demonstrated positive physiological and behavioral outcomes as well as incentive driven outcomes with mHealth systems [14-17]. However, there is limited literature showing that mHealth approaches can be useful for the self-management in patients with other chronic diseases. Therefore, an indepth evaluation of RCTs on interventions that employ mHealth technologies and participants' adherence to the interventions, training methods, intervention dosage, and length of follow-ups as outcomes of interest should be performed to provide recommendations on what factors make mHealth interventions effective for chronic disease management.

Thus, the purpose of this study was to perform a systematic review of RCTs using mHealth interventions for chronic disease management in adult populations to examine the effectiveness of mHealth interventions on health outcomes and process measures.

\section{Methods}

The Preferred Reporting Items for Systematic Review and Meta-Analysis (PRISMA) guidelines [18] were used in this systematic review. The PICOS (participants, interventions, comparisons, outcomes, and study design) approach was used to develop a research question to guide the search strategies and review: that is, do interventions using mobile health applications improve health outcomes and process measures for adults with chronic diseases in RCTs?

\section{Search strategies}

Searches were performed to retrieve studies that were published in peer-reviewed journals from January 2005 to March 2016, and written in English; the following databases were used: PubMed, CINAHL, EMBASE, the Cochrane Library, PsycINFO, and Web of Science. We used combinations of the key words and indexing terms such as MeSH or Emtree linked to the search domains. An example of a PubMed search strategy is as follows: for mobile interventions, "Mobile Applications"[Mesh] OR "Cell Phones"[Mesh] OR "Computers, Handheld"[Mesh] OR "mobile health" OR "m-health" OR mhealth OR "mobile-health" OR smartphone* OR "smart-phone*" OR "mobile phone" OR "mobile-phone*" OR "cellular phone $^{* \text { " OR "cellular-phone }}$ "* OR "smart device" "smart-device" "tablet" device";"; for chronic disease outcomes, "Disease Management"[Mesh] OR "Chronic Disease/prevention and control"[Mesh] OR "Chronic Disease/therapy"[Mesh] OR "disease* manag*" OR "disease" monitor" OR monitor* OR "health promot*" OR Promot*, and for method, "Randomized Controlled Trial" [Publication Type] OR "Randomized Controlled Trials as Topic"[Mesh] OR "Controlled Clinical Trial"[Publication Type] OR randomized[Title/Abstract] OR randomised[Title/Abstract] OR randomly[Title/Abstract] OR "random" assign""[Title/Abstract] OR trial*[Title/Abstract]. Then, those three groups of search results were combined with "AND" (see Additional file 1 for search strategy for each database).

\section{Study selection}

The inclusion criteria were as follows: adult patients with chronic diseases (except diabetes) as the target population, an intervention that involved using a mobile application for smartphones or tablets, and assessing the health outcomes and process measures. The exclusion criteria were as follows: studies that focused on a healthy population, pregnant women, non-adults (i.e., adolescents and children), or healthcare providers (e.g., apps 
for physicians' or nurses' use only); studies that used only qualitative methods (e.g., focus groups or group/individual interviews) or simple usability tests; and studies that measured psychological outcomes only (Table 1). Two reviewers (MC and SAL) independently screened titles, abstracts, and full-text articles to decide whether an article was relevant to the review. In case of disagreement, a third person was consulted (JL). We excluded studies of diabetes management because several systematic reviews and integrated reviews have already been published to report the effectiveness of mHealth-based interventions [14-17]. Only studies published in peerreviewed journals were included.

\section{Data extraction}

Data were extracted from the selected articles and entered into an electronic data sheet. The contents of the data sheet included year of publication, research question or purpose, study design, types of disease, types of outcome and measurement, and the main results. In instances of disagreement, each case was discussed by the authors.

\section{Assessment of risk of bias}

Selection bias (random sequence generation and allocation concealment), performance bias (blinding of participants and personnel), detection bias (blinding of outcome assessment), attrition bias (incomplete outcome data), reporting bias (selective reporting), and other biases (determined according to sample size calculation method, inclusion/exclusion criteria for patients' recruitment, comparability of baseline data, funding sources, and any other potential methodological flaw that might have influenced the overall assessment) were assessed with the tool for risk of bias given in the Cochrane Handbook for Systematic Reviews of Intervention [19]. For each risk of bias item, the studies were classified as "unclear," "low," or "high" risk of bias respectively. Two reviewers (MC and SAL) assessed the

Table 1 Inclusion and exclusion criteria

\begin{tabular}{ll}
\hline $\begin{array}{l}\text { Inclusion } \\
\text { criteria }\end{array}$ & - studies that included adult patients with \\
& chronic diseases (except diabetes) as the \\
& target population \\
- studies that involved using a mobile application & - studies that focused on disease management \\
& or health promotion \\
Exclusion & - studies that included healthy people, pregnant \\
criteria & women, non-adults (i.e., adolescents and children), \\
& and healthcare providers \\
& studies that used only qualitative methods \\
& or simple \\
& usability tests \\
& oudcomes only \\
\end{tabular}

trials independently and disagreements between two authors were resolved via discussion.

\section{Operational definitions of the terms used in this review}

Studies on feasibility assess whether or not an intervention is appropriate for further testing, whereas studies on acceptability (which is a component of feasibility) determine how recipients react to that intervention [20]. Effectiveness is defined as an intervention study that shows statistical differences of one or more outcomes of interest measured between intervention and control groups. Health outcomes included physiological outcomes (e.g., gait and balance in patients with Parkinson's disease or fatigue in patients with cancer) and psychological outcomes (e.g., quality of life, depressive symptoms, anxiety). Process measures [21] included participants' adherence to, satisfaction with, and/or the level of engagement with mHealth systems. These process measures could be assessed via quantitative tools such as surveys or qualitative methods such as openended questions or a focus group interviews with intervention participants.

\section{Results}

Figure 1 shows the PRISMA flow diagram indicating the search process to select the final studies that met the inclusion criteria and thus were included in this systematic review. A study conducted by Kristiánsdóttir et al. was published as part 1 [22] and part 2 [23], which corresponded to short-term and long-term follow-ups, respectively. The results of both follow-ups were reviewed. Accordingly, the results of this systematic review are based on 12 studies from 13 published articles with quantitative evaluations.

Table 2 presents the summary of 12 RCT studies reviewed in this paper. The variety of chronic diseases managed using mobile apps included allergic rhinitis and asthma, cancer, cardiovascular diseases, chronic pain, chronic kidney disease, lung transplantation, Parkinson's disease (PD), and spinal bifida. Diabetes management using mHealth apps has been evaluated in other literature and thus was not included. Among the 12 studies reviewed, 10 studies showed statistically significant mHealth app intervention effects on some variables that were examined in each study, while two of the 12 studies reviewed showed no statistically significant mHealth intervention effects on outcomes of interest when comparing between treatment and control groups. Two studies in this review were feasibility studies aimed at testing mHealth interventions for chronic disease management $[24,25]$ and one study was a pilot study evaluating a smartphone-based symptom management system for chemotherapy management [26]. These studies were also listed as RCTs. 


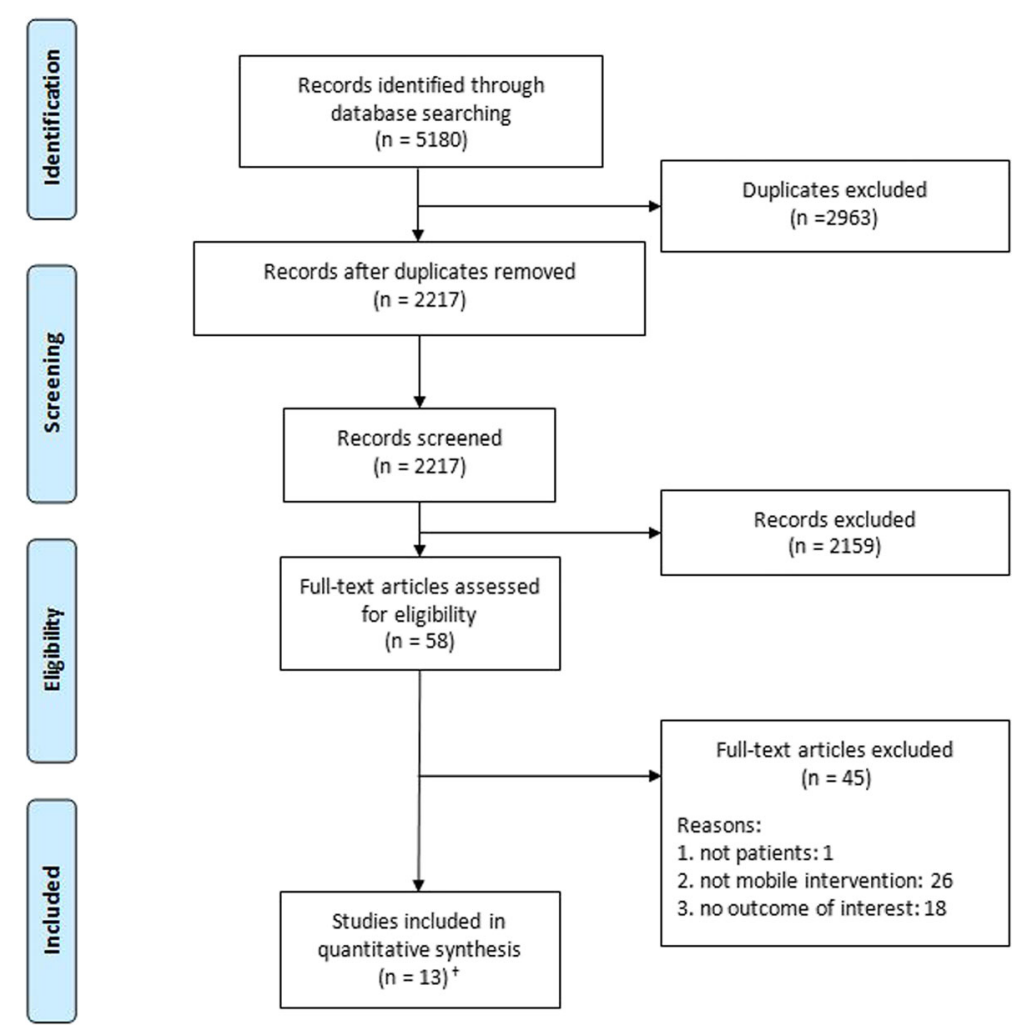

Fig. 1 PRISMA flow diagram for the systematic review process. The step-by-step process of the application of inclusion and exclusion criteria generated the final number of studies included in the systematic review. ${ }^{\dagger}$ Note: Kristjánsdóttir et al. (2013) was published as part 1 [22] and part 2 [23] with respect to a short-term follow-up and long-term follow-up; thus, the results are based on 12 studies from 13 published articles

\section{Studies reporting significant effects on outcomes}

The majority of mHealth RCT-studies in this systematic review (10 out of 12 studies, 83.3\%) showed statistically significant effects on health outcomes by incorporating mobile applications in managing chronic diseases. Those studies demonstrated improved physical functioning, adherence to prescribed medications, and/or ease of symptom evaluation and reports to care providers, as well as process measures including patient satisfaction with mHealth management and feasibility of smart-phonebased self-management interventions.

Kearney et al. [26] in the United Kingdom reported significant improvement in fatigue (odds ratio, $\mathrm{OR}=$ 2.29; 95\% CI, 1.04-5.05; $P=0.040$ ) and hand-foot syndrome (OR control/intervention $=0.39$; 95\% CI, 0.17 $0.92 ; P=0.031)$ in patients with lung, breast, and colorectal cancer using a mobile phone-based remote monitoring of chemotherapy-related symptoms in comparison to the usual care group. In Norway, Kristjánsdóttir et al. [22, 23] showed a favorable effect on pain management in a 4-week follow-up (catastrophizing score lower for Intervention, $M=9.20, \mathrm{SD}=5.85$, compared to Control, $M=15.71, \mathrm{SD}=9.22, P<0.01$, with a large effect size, Cohen's $d=0.87$ ) but not in the 5month and 11-month follow-ups (outcome variables including catastrophizing, acceptance, functioning, and symptom level, all $P>0.1$ ). In Spain, Garcia-Palacios et al. [27] developed an ecological momentary assessment (EMA) for chronic pain in fibromyalgia patients and found that patients with less familiarity with technology using a mobile EMA system via their smartphones showed higher levels of compliance than patients with a paper-based diary (complete record $t=-4.446, d=1.02$, reference Cohen's $d>=0.8$, large effect). In Turkey, Cingi et al. [28] reported that patients with allergic rhinitis or asthma displayed better quality of life or well-controlled asthma scores by using the mHealth intervention compared to the control group (all $P<0.05$ ). Dicianno et al. [24] demonstrated the feasibility of a mHealth intervention for patients with spina bifida to improve selfmanagement skills and high usage of the mobile system was associated with positive changes in the selfmanagement skills. In Sweden, Hägglund et al. [29] tested a tablet-based intervention in patients with heart failure (HF) and found improved self-care and healthrelated quality of life (HRQoL) and a reduction in HFrelated hospital days (risk ratio, $\mathrm{RR}=0.38$; 95\% CI, 0.310.46; $P<0.05)$. In Israel, Ginis et al. [25] conducted home-based smartphone-delivery automated feedback training for gait in people with PD, and found significant 


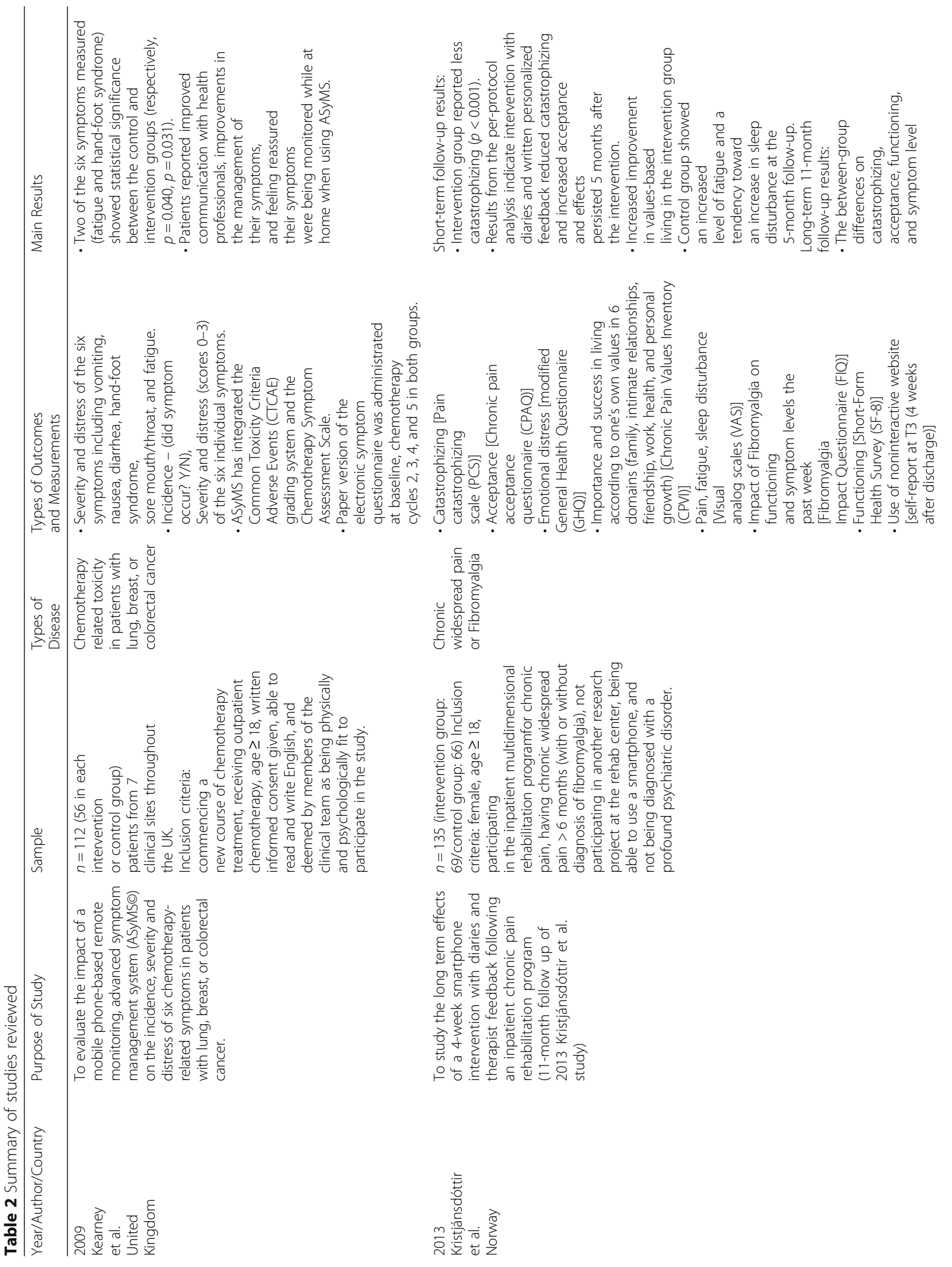




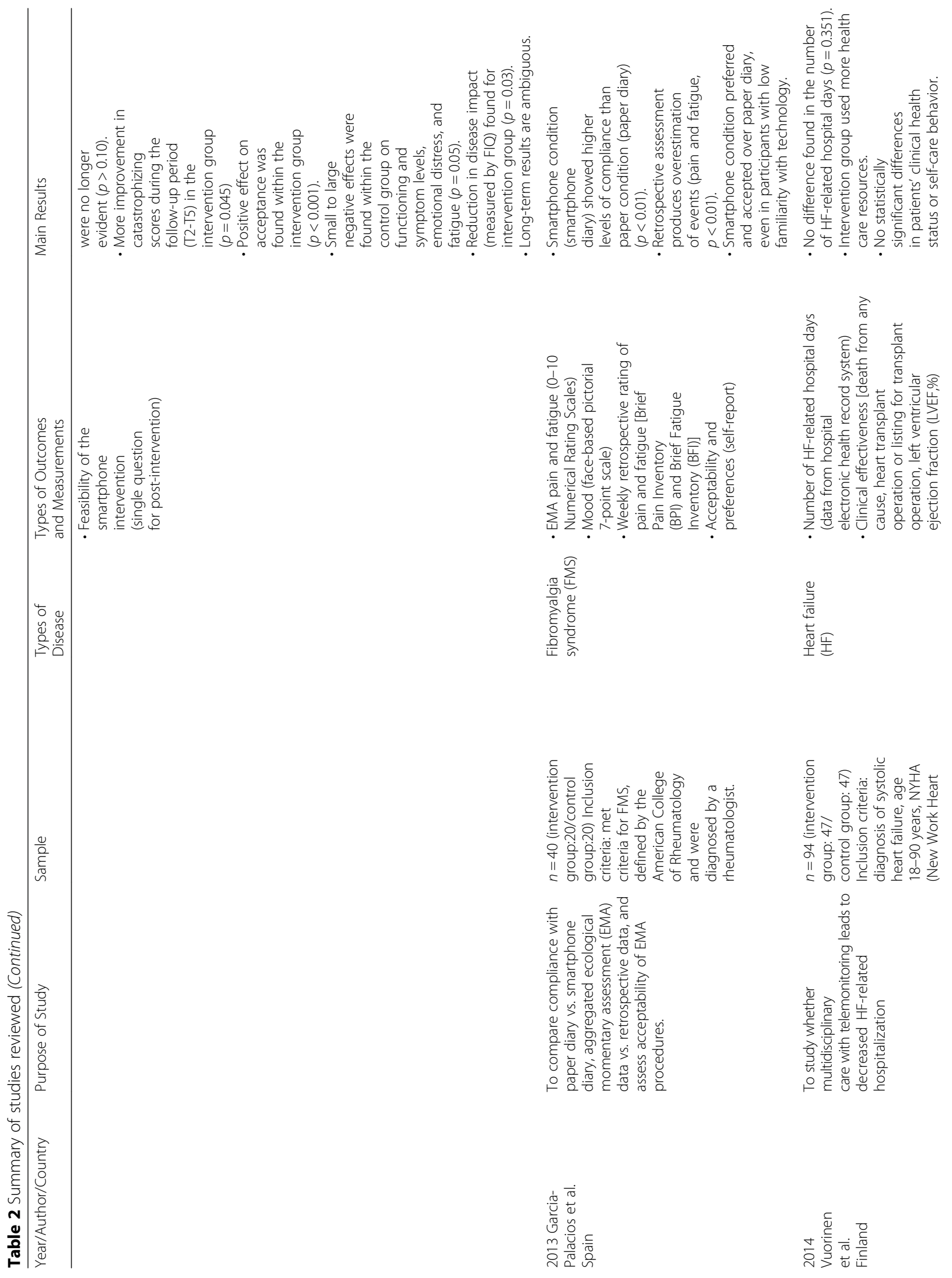




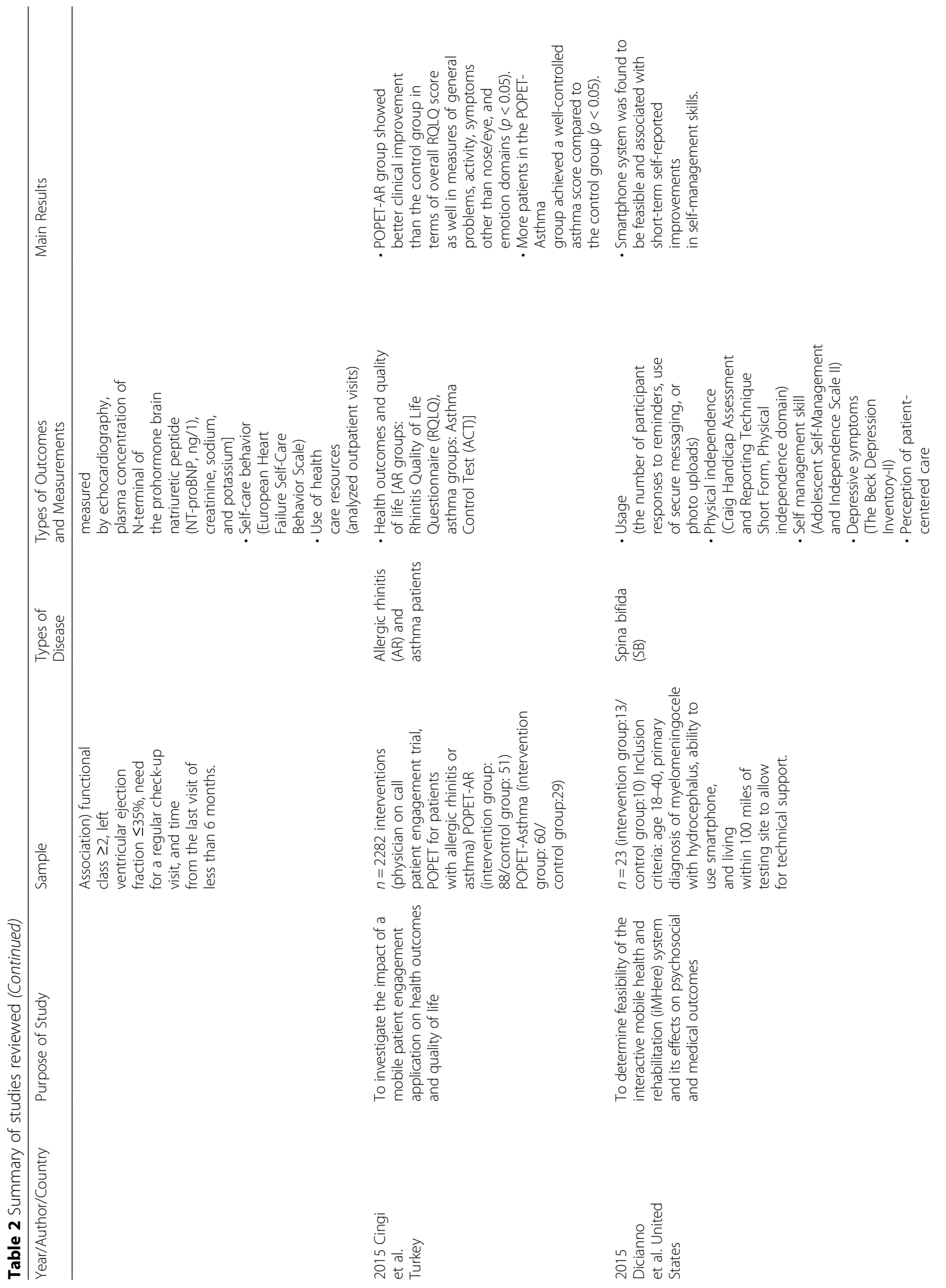




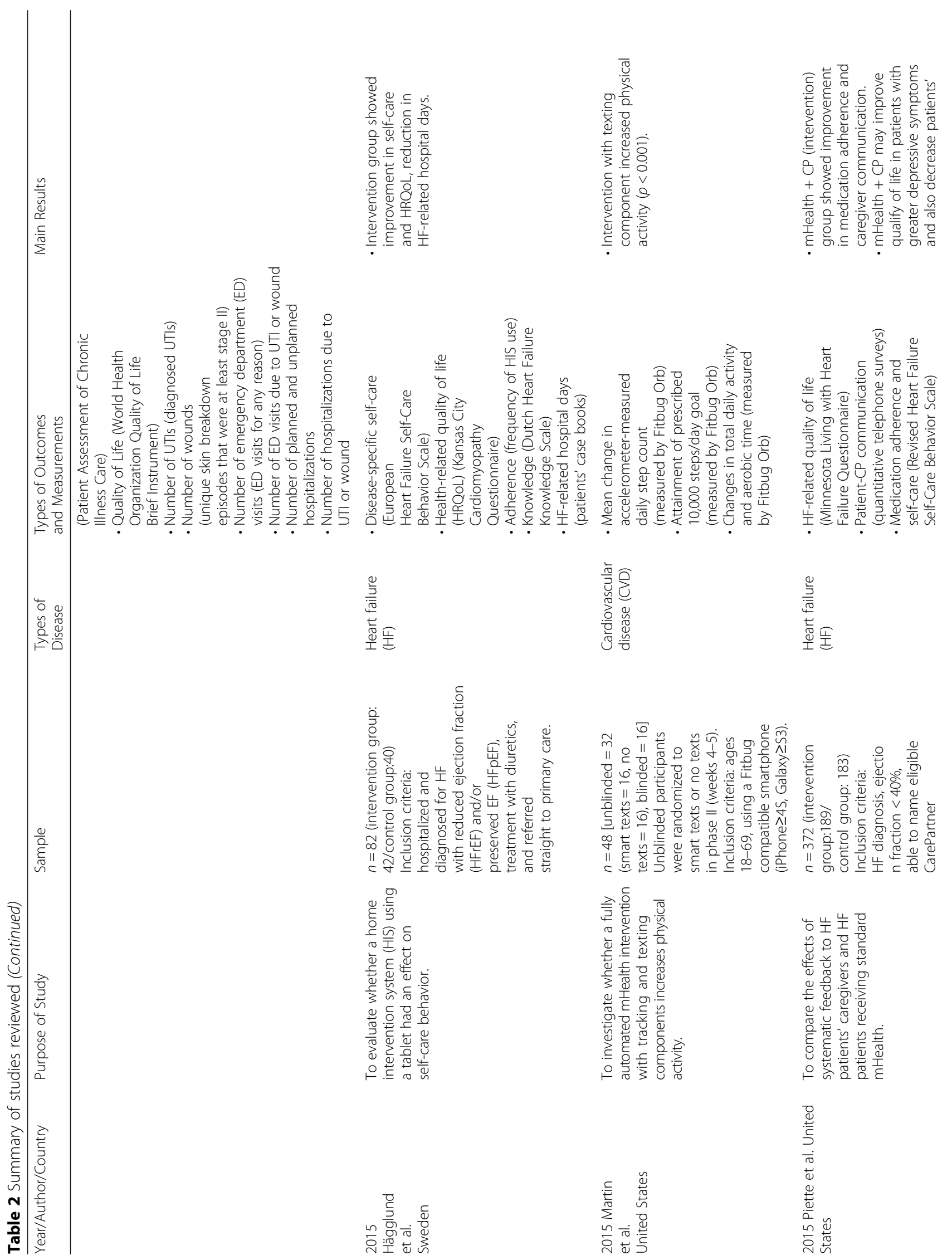




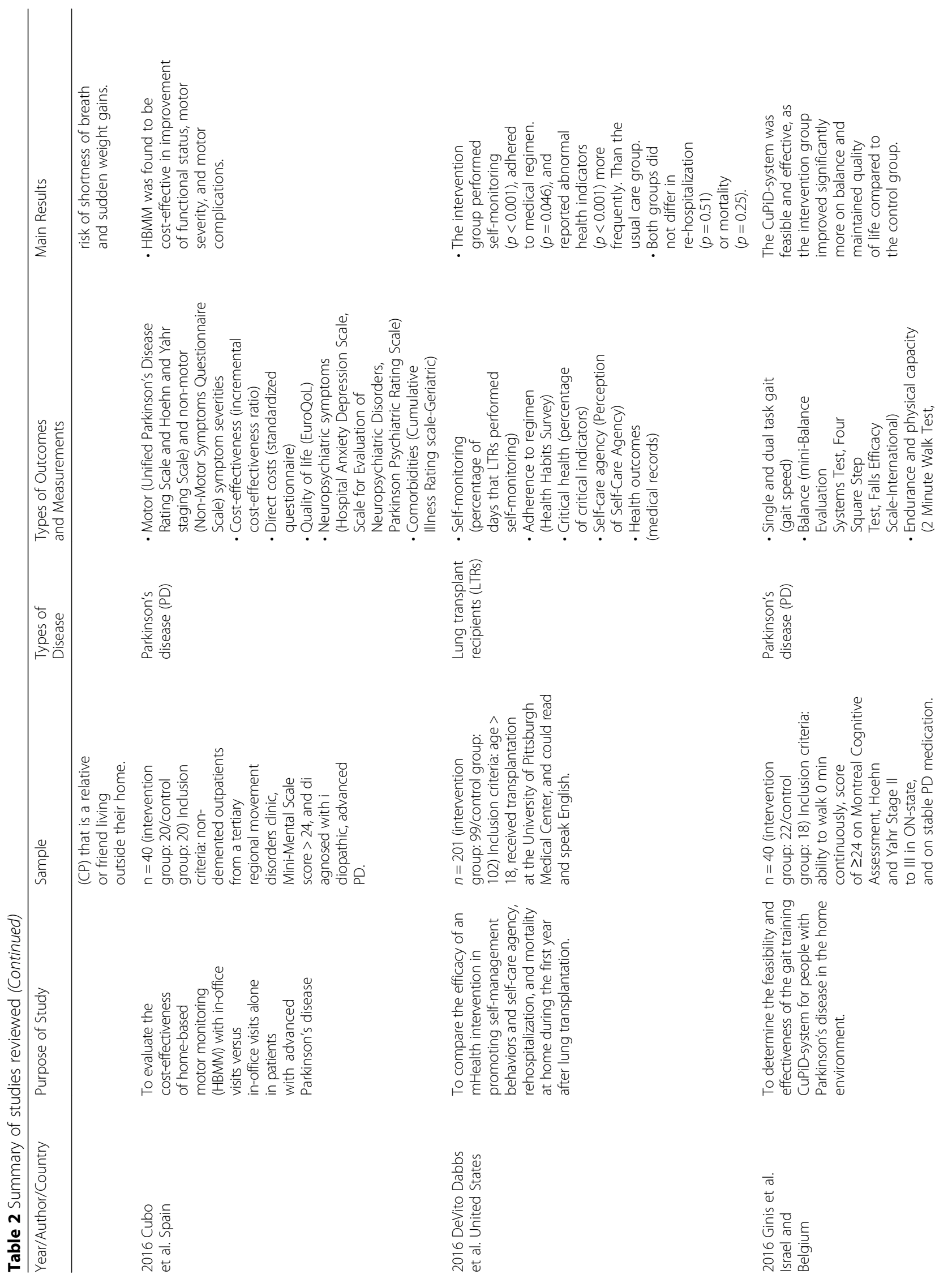




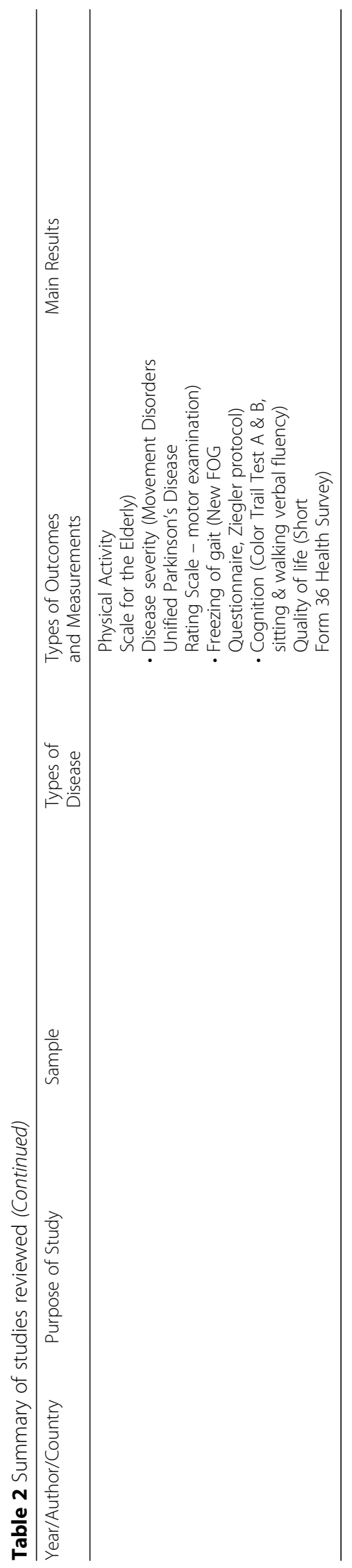


improvement in balance $\left(F_{(2,108)}=3.73, P=0.04\right)$ from baseline to post-test.

Martin et al. [30] in the US found that an automated text message system increased physical activity to prevent cardiovascular diseases in phase 1 (weeks 2 to 3 ) and phase 2 (weeks 4 to 5), all $P<0.001$. Piette et al. [31] in the US reported that the intervention group involving "CarePartners" connecting to a relative or friend living outside their home showed improvement in medication adherence and caregiver communication (all $P<0.05$ ). DeVito Dabbs et al. [32] in the US conducted an RCT for patients with lung transplantation and reported improvement in self-monitoring $(\mathrm{OR}=5.11$; 95\% CI, 2.958.87; $P<0.001$ ), adherence to medical regimen (OR $=1.64 ; 95 \% \mathrm{CI}, 1.01-2.66, P=0.046)$, and reported abnormal health indicators more frequently $(\mathrm{OR}=8.9 ; 95 \%$ CI, 3.60-21.99; $P<0.001)$.

\section{Studies reporting similar or no effects on outcomes}

Two of the twelve studies reviewed (16.7\%) showed similar or no effects of mHealth-based interventions on main outcomes of interest compared to control groups. In Finland, Vuorinen et al. [33] found no difference in the number of HF-related hospital days (incidence rate ratio, $I R R=0.812, P=0.351$ ). However, patients in the telemonitoring intervention group used more healthcare resources; increased number of visits to the nurse (IRR $=1.73 ; 95 \% \mathrm{CI}, 1.38-2.15 ; P<0.001)$, more time spent with nurses (mean difference $=48.7 \mathrm{~min}, P<0.001$ ), and increased number of telephone contacts initiated by nurses $\quad(\mathrm{IRR}=5.6 ; 95 \%$ CI, 3.41-7.63; $P<0.001)$. In Spain, Cubo et al. [34] reported a trend of lower PD functional status (the Unified Parkinson's Disease Rating Scale, UPDRS I) in patients on home-based monitoring compared to patients in standard in-office visits $(P=$ 0.06), while other outcomes (measured by UPDRS II, III, IV subscales) and HRQoL in PD did not show statistically significant differences between the intervention and control groups. Cubo et al. [34], however, explained that the approach of using home-based motor monitoring via mHealth applications compared to standard office visits in the 1-year follow up was cost-effective (incremental cost-effectiveness ratio, ICER, per unit of UPDRS subscales ranging from $€ 126.72$ to $€ 701.31$ ).

\section{mHealth interventions}

Table 3 presents the details of mHealth interventions including duration, mobile app type, app content, and training methods. A quarter of the studies (3 out of 12) reported the feasibility of the mHealth intervention as a pilot study to assess the potential for successful implementation of the mHealth intervention to patients/participants [24-26]. The majority of studies used smartphones as a mobile device, two studies
[29, 34] used tablets for mHealth interventions, and two studies used telemonitoring wireless devices including weight scales for patients with HF [29] or gait detectors for patients with PD [25]. The length of interventions ranged from two weeks to twelve months; half of the studies $(6 / 12,50 \%)$ had more than sixmonth intervention periods and follow-ups. Most common components of mHealth interventions included remote symptom monitoring and selfassessment as well as tailored automated messages or self-care education to coach patients with chronic disease conditions that needed active disease management. One particular study by Kearney et al. was more inclusive in that it provided real-time feedback and tailored such feedback for symptom management depending on the severity, offering pharmacological, nutritional, or behavioral advice when needed [26].

The sample size of the studies reviewed was between 28 (patients with spina bifida) and 372 (patients with heart failure). In terms of subjects, studies included patients who were 18 years and above, with some studies limited to a particular age range such as 18-40 [24] or up to 69 years [30]. The mean age of participants in the mHealth intervention studies ranged from 30 -year-old patients with spina bifida [24] to 75-year-old patients with HF [29]; the approximate average age group in this 12-study review was in the $50 \mathrm{~s}$. None of the studies reported effectiveness of mHealth intervention by age categories. These 12 studies also did not report participants' prior experience with mobile devices such as smartphones or educational background, which may have affected the ability to use mHealth apps via mobile devices. Outcomes of interventions were measured either by mHealth systems directly or by paper-based questionnaires.

In terms of mHealth intervention training, either face-to-face information sessions at baseline or paperbased instructions were used in most studies. Kearney et al. [26] found that symptoms were reported differently on a paper-based questionnaire and mobile phone. Participants in the mobile group reported lower levels of fatigue compared to those in the paper-based group (OR non-mobile $/$ mobile $=2.29,95 \% \mathrm{CI}$ 1.04-5.05, $P=0.04$ ) [26]. Reporting cancer toxicity symptoms (e.g., hand-foot syndrome and mucositis) in real time might allow for more accurate measurement [26].

None of the studies reported process measures, including adherence, level of engagement, and/or satisfaction with the mHealth systems. Potentially, there was limited information on such measures in the published articles with the authors possibly publishing the process measures elsewhere. 


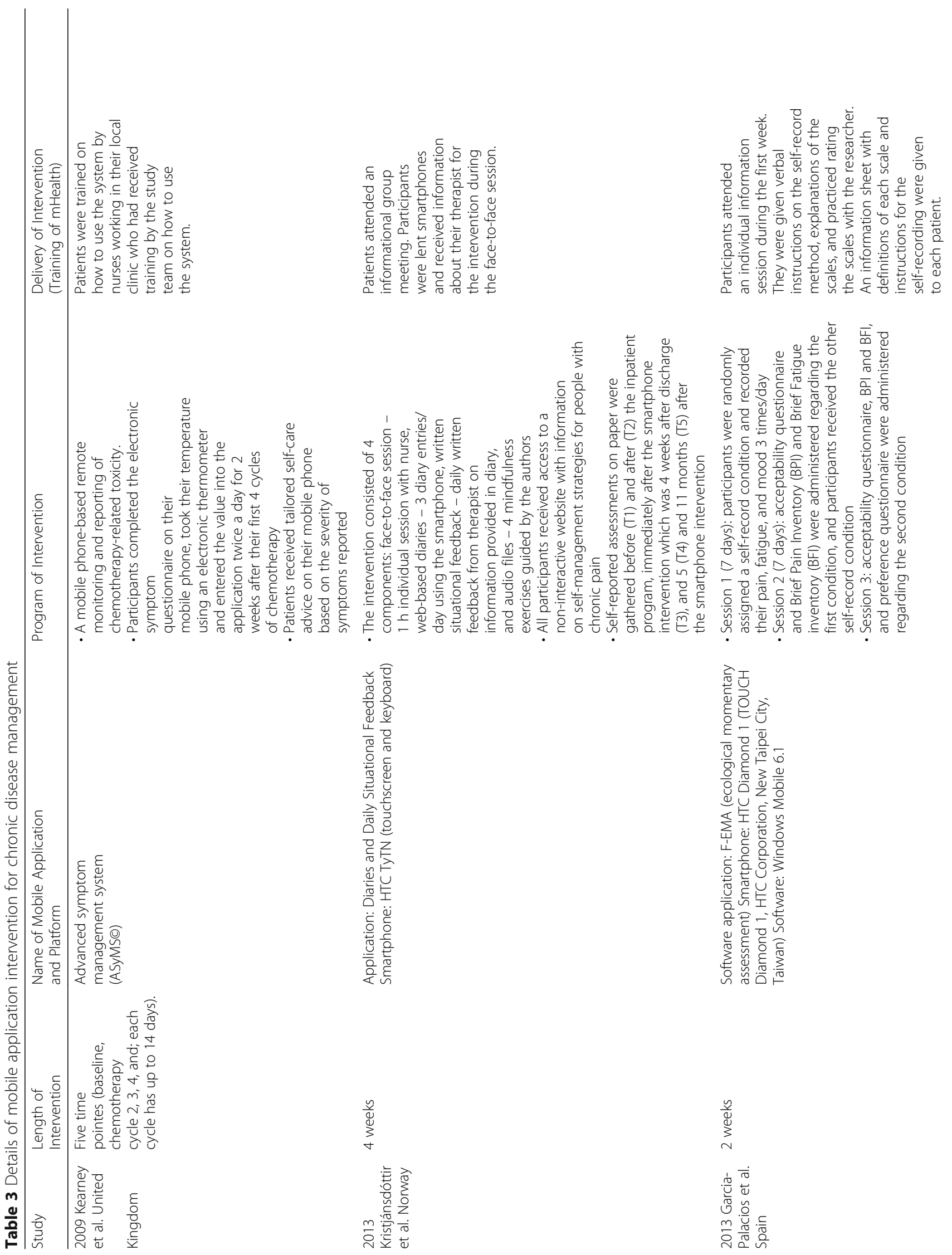




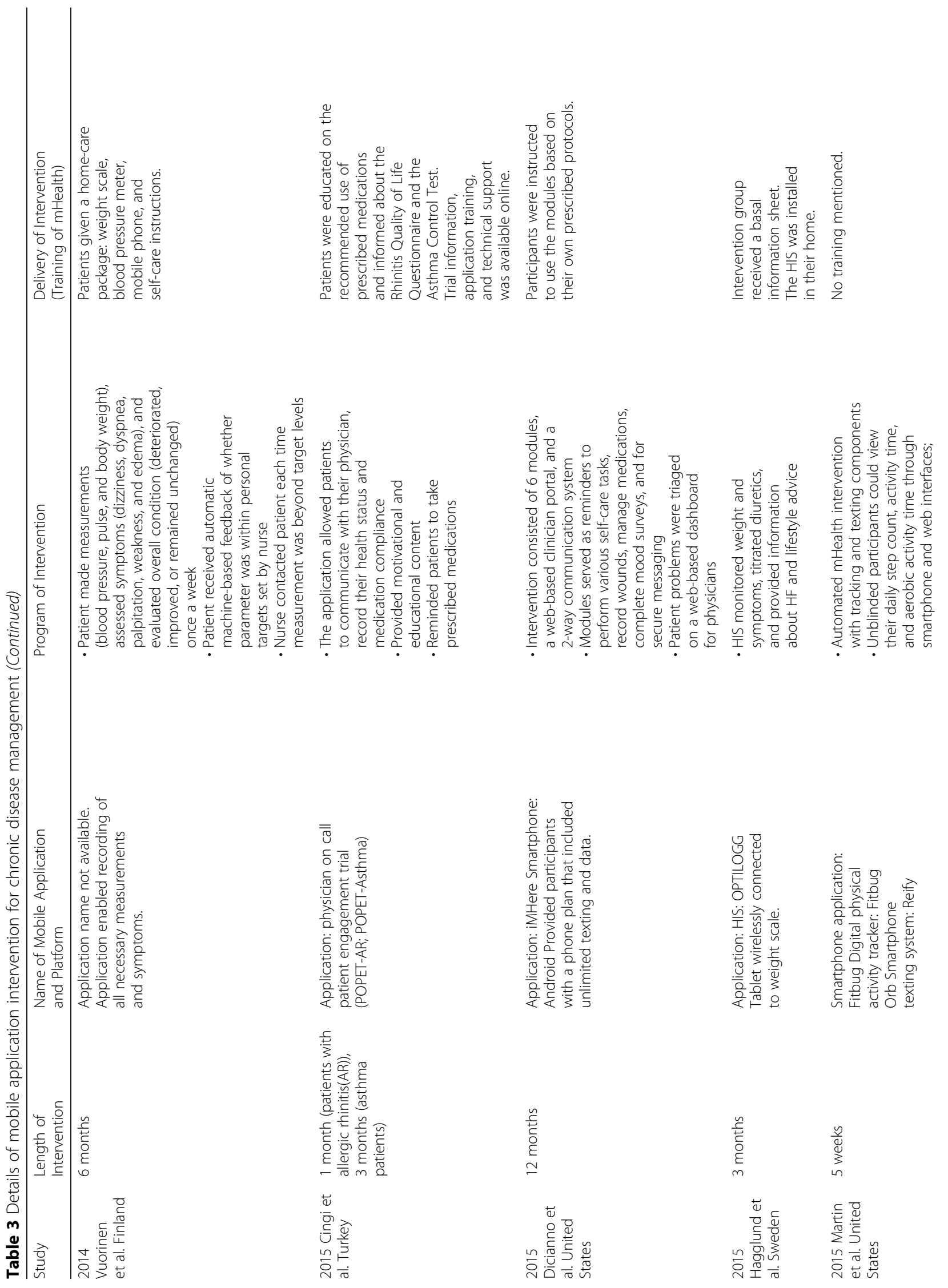




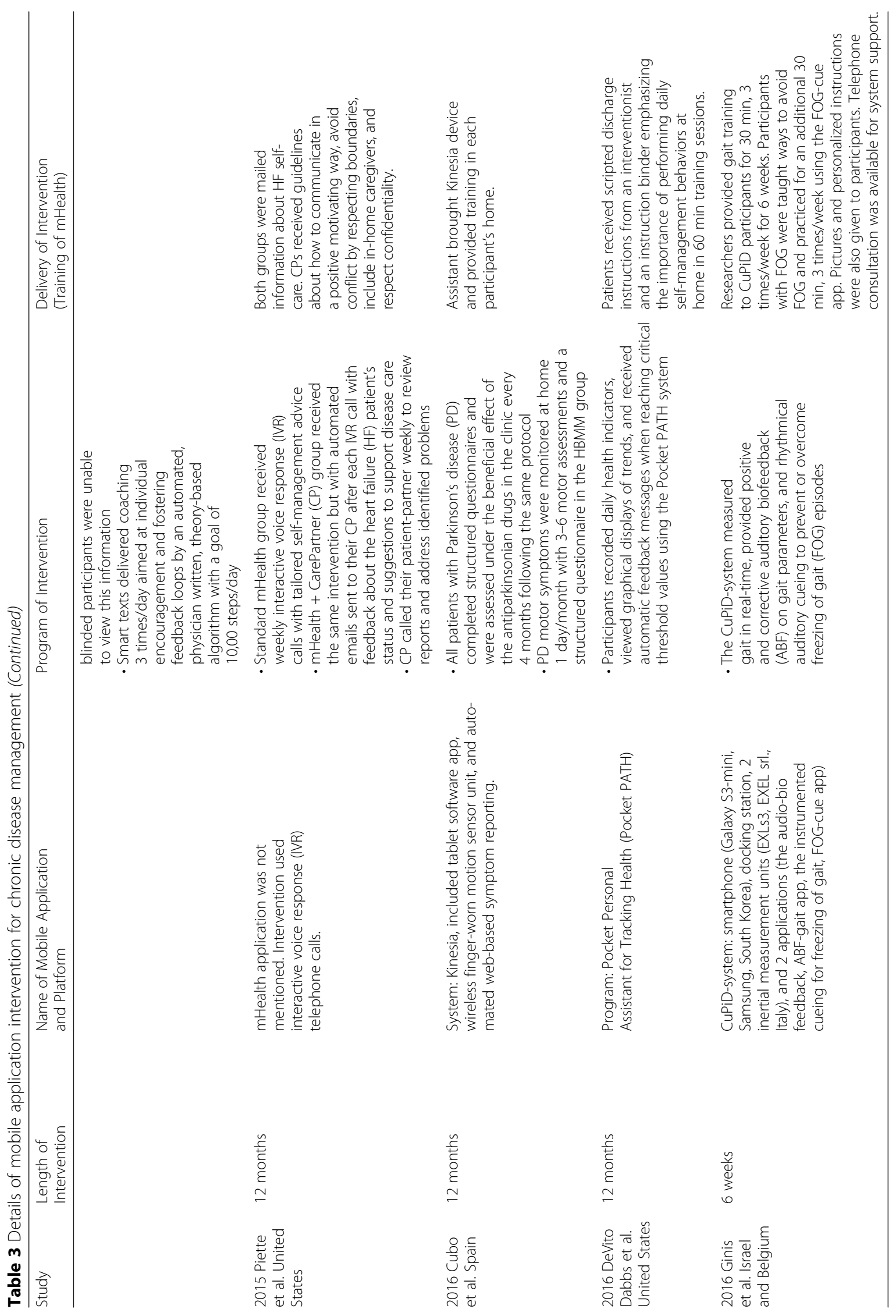




\section{Risk of bias assessment}

The risk of bias in the reviewed studies is summarized in Fig. 2 and shown for individual studies in Fig. 3. The intervention studies generally performed well in their risk of bias for random sequence generation (62\% low risk), allocation concealment (23\% low risk), blinding of participants and personnel (8\% low risk), blinding of outcome assessors (15\% low risk), incomplete outcome data (69\% low risk), and selective reporting (92\% low risk) (Fig. 2).

Considering the method of randomization, 5 trials did not present details of randomization [25, 27, 29, 30, 33]. Only 2 studies reported use of an adequate allocation concealment method [22, 32]. Although due to the nature of the mHealth intervention, it is almost impossible to blind participants and healthcare providers, one study described the blinding of participants by providing the same mobile phone application to both experimental and control groups with different functionalities. The experimental group was given communication, health status, or medication usage tracking, and a survey questionnaire, whereas the control group received only the survey questionnaire [28]. Only two trials were identified as blinding the outcome assessors. Four trials failed to provide a full description of participants and losses to follow-up during their trials [24, 28, 29, 31]. All studies had a low risk for reporting bias. Devito et al. had low risk for all items except blinding of participants and personnel [32]. Hägglund et al. had low risk only for reporting bias [29] (Fig. 3).

\section{Discussion}

This systematic review has found a potential favorable effect of mHealth interventions on health outcomes and process measures in patients with chronic diseases including asthma, cancer, cardiovascular diseases, chronic pain, spina bifida, or Parkinson's disease. The results from the reviewed RCTs showed improvement in some health outcomes in patients in managing their chronic disease.

There were some commonalities and differences in using mHealth in the reviewed studies. One of the common features useful in mHealth interventions is pre-set and tailored feedback on reported symptoms. The mobile application systems used in the reviewed studies were developed by the study team and validated and refined in the previous studies that were conducted before the RCTs. None of the commercial health apps were used in the reviewed studies. Most studies utilized research staff to provide training in mHealth systems for the participants via face-to-face or information group sessions, or through information materials, while one study [26] used local nurses to train patients. None of the reviewed studies addressed whether their mHealth systems were incorporated into daily medical practice in either clinic settings or acute care hospitals, meaning that there were no signs of their implementation in real health care systems. Challenges in real-life settings may relate to lack of financial incentives for providers in using mHealth tools or uncertainty regarding privacy and security of information transferred via mHealth systems [35].

Interventions to promote self-management in patients with chronic diseases started from web-based and/or telephone-based interventions to mHealthbased interventions. Unlike those previous behavioral interventions limited to places where patients with chronic diseases had access to the treatment advice, mHealth interventions have advanced features such as real-time symptom monitoring and feedback [25, 34, 36]. For example, patients with $\mathrm{PD}$ receive real-time feedback on their selected gait parameters during their walks via the preset gait app developed from evidence-based exercise guidelines [25]. This is an example of how using well-designed and validated mHealth apps in daily life can benefit health outcomes.

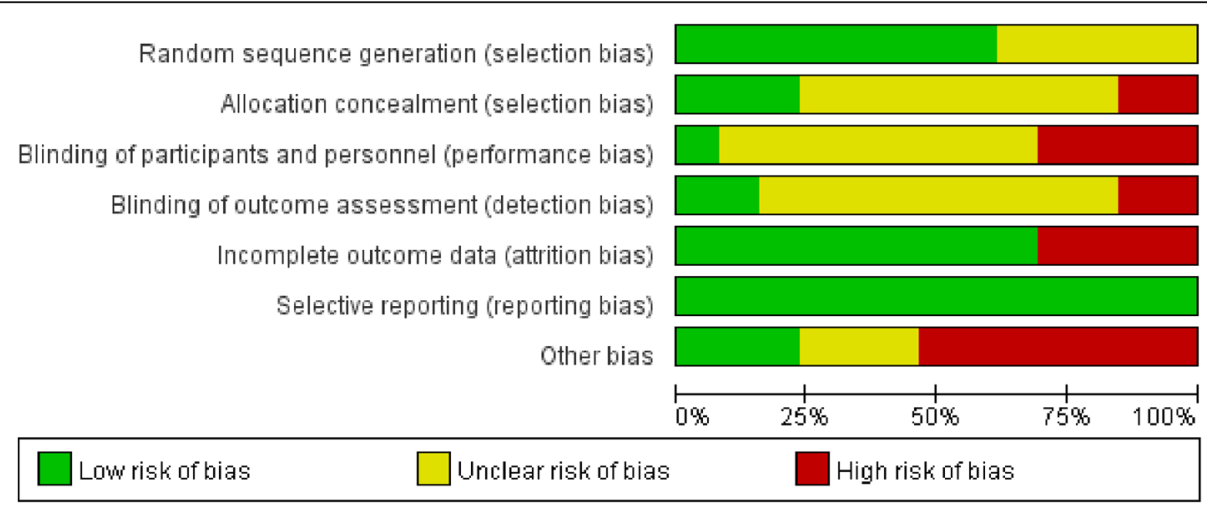

Fig. 2 Risk of bias assessment: Summary graph 


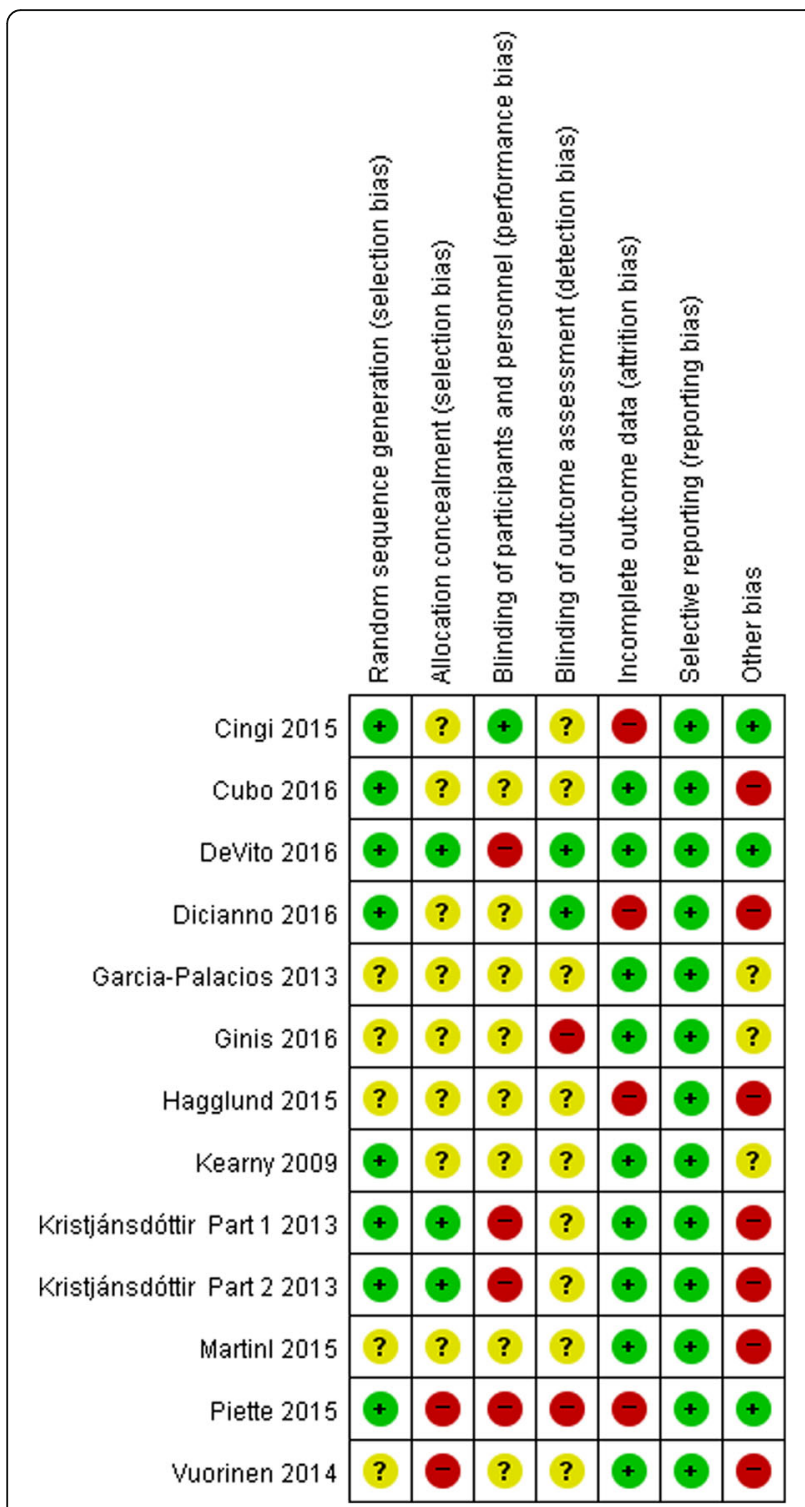

Fig. 3 Risk of bias assessment for individual studies. + Low risk of bias; ? Unclear; - High risk of bias

The number of smartphone users has shown great increases; in the US [37] it is estimated to reach 224.3 million in 2017, up from 171 million in 2014; worldwide it was [38] 2.32 billion in 2017, up from 1.57 billion in 2014. Approximately $77 \%$ of people in the US owned smartphones in 2016 [39]. Using mobile devices for mHealth is essential nowadays and approaches of mHealth vary from sending text messaging for medical appointment reminders to monitoring and assessing symptoms in real-time, virtually at any location via wireless networks. Interventions using mHealth have also eased medical coaching for caregivers as care partners with healthcare professionals for effective chronic disease management. Piette et al. [31] studied the comparative effectiveness of mHealth interventions supporting HF patients and their family caregivers and showed improvement for medication adherence and caregiver communication. The impact of including caregivers as a part of mHealth users-one of the care supporting groups-on actual patients' health outcomes should continue to be studied in the context of an increasing aging society.

Moreover, long-term follow-ups of responses from patients and caregivers who have used mHealth need to be evaluated. Areas that need to be studied include the optimum length of time and frequency of the mHealth delivery system as well as type of technology and training. For example, effective frequencies of automated reminders or coaching messages, when additional reminders should be sent, and when people become tired or irritated by automated messages need to be studied. Users of mHealth might experience fatigue from automated reminders and eventually mHealth interventions could become ineffective. Other systematic reviews on chronic disease management showed that the frequency of input into mHealth systems was a burden on participants and affected the attrition rate [17]. In this review, the length of the intervention in the 12 studies varied from 2 weeks to 12 months; 5 of 12 studies (about $42 \%)$ had less than 2-month interventions while 4 studies (about 33\%) had 1 year of intervention. The positive health outcomes of the various studies were not directly related to the length of the intervention or training methods of mHealth in the reviewed studies.

One aspect of mHealth approaches that also needs to be considered is effective clinical communication between patients and healthcare professionals who need to respond to patients' questions via mHealth systems. Cingi et al. [28] reported healthcare providers (i.e., residents) expressed an improvement in communication with patients via mHealth; however, they found that using mHealth tools as the primary method of communication was strongly opposed by the healthcare providers. Vuorinen et al. [33] also reported a significant increase in the communication (i.e., telephone contacts) between nurses and patients which in turn increased the nurses' workload during the trial. One recommendation for reducing health care providers' workload in mHealth interventions is using advanced technology to respond to patients' questions regarding symptoms assessed and reported via the mHealth systems.

While considering positive health outcomes (e.g., reduction of hospital readmission for HF-related conditions or medication adherence) of patients with chronic diseases, burden of healthcare professionals should be measured as an outcome of mHealth interventions. An adequate triage system can decrease healthcare 
professionals' response time for emergency needs reported by mHealth users [28].

Common recommendations discussed in the studies of this systematic review to improve mHealth interventions include a simple and user-friendly-designed mHealth system, data confidentiality, lay language use for structured and automated feedback or advice, positive motivation and improving engagement [28], and inclusion of patient's social supporters, such as family members, friends, and/or peers [31].

There are several limitations in this systematic review. First, we only selected randomized controlled trials for this review and most of them were funded studies. Therefore, the mHealth systems or smartphone apps used in the studies were validated and relatively reliable compared to health apps commercially available on the market. Literature from studies on smartphone-based interventions for chronic disease management that were small scale with or without control group and/or had a short-term follow-up has shown ambivalent results. Thus, we intended to choose robust studies that used randomization, control groups, and relevant follow-ups for outcomes. We looked at the outcome changes at different follow-up points.

Second, we did not include mHealth intervention studies for diabetes management since there is ample literature on diabetes management using mobile technology approaches [14-17]. Third, we excluded studies wherein health apps were used only by health professionals such as physicians or specialized clinical nurses. We focused on patient-centered health apps as a part of mHealth interventions in the review. Lastly, most of the reviewed studies provided smartphones or tablets to participating patients and thus, the results from this review cannot yet be generalized among those who have financial concerns regarding purchasing mHealth tools. Although the availability of wireless networks is increasing, potential mHealth users such as patients with chronic diseases or their caregivers may have limited data services for their mobile devices due to financial concerns. This might be an important issue during an emergency when patients may have no access to evidence-based medical advice via mHealth devices.

\section{Conclusion}

The findings from the majority of reviewed studies that used mHealth interventions showed some health outcome improvement in patients with chronic disease conditions. Favorable factors in mHealth approaches are automated text reminders, frequent and accurate symptom monitoring (often in real time), and improved communication between patients and healthcare providers resulting in enhanced self-management in patients with chronic conditions. Thus, the future of mHealth is presumably optimistic. The relationship between engagement of users on mHealth tools and outcome improvement should be further studied. The studies reviewed in this paper showed disease-specific mHealth interventions that might be different from commercial mobile health apps available to the public. Rigorously tested mHealth apps developed through research should be further considered to be made available to the general population.

\section{Additional file}

Additional file 1: Title of data: Detailed search strategy per database. Detailed search strategy per database in order to find all published interventions using mobile health applications to improve chronic disease management for adults in randomized controlled trials. (DOCX $21 \mathrm{~kb}$ )

\section{Abbreviations}

CDC: The US Centers for Disease Control and Prevention; EMA: Ecological momentary assessment; HF: Heart failure; mHealth: Mobile health;

PD: Parkinson's disease; RCTs: Randomized controlled trials; UPDRS: Unified Parkinson's Disease Rating Scale; US: The United States of America

\section{Acknowledgements \\ The authors thank Yoori Yang, MSN, College of Nursing, Yonsei University and Laura Narvaez, BS, Sue and Bill Gross School of Nursing at University of California Irvine for their assistance in literature search and abstract reviews. We also thank Dr. Priscilla Kehoe for her thorough editorial support.}

Funding

Not applicable.

Availability of data and materials

Not applicable.

\section{Authors' contributions}

Design of the study protocol: JL, MC; Data collection and analysis: MC, SAL, $\mathrm{NJ}$; Data interpretation: JL, MC, SAL; Drafting the manuscript: JL, MC, SAL, NJ. All authors revised the article critically, gave feedback and approved the final manuscript.

Ethics approval and consent to participate Not applicable.

Consent to publication

Not applicable.

Competing interests

The authors declare that they have no competing interests.

\section{Publisher's Note}

Springer Nature remains neutral with regard to jurisdictional claims in published maps and institutional affiliations.

\section{Author details}

${ }^{1}$ Sue and Bill Gross School of Nursing, University of California Irvine, Irvine, CA, USA. ${ }^{2}$ College of Nursing, Mo-Im Kim Nursing Research Institute, Yonsei University, 50 Yonsei-ro, Seodaemun-gu, Seoul, Republic of Korea03722.

${ }^{3}$ College of Nursing and Health Sciences, University of Massachusetts, Boston, MA, USA. ${ }^{4}$ Program in Public Health, University of California Irvine, Irvine, CA, USA. 
Received: 24 June 2017 Accepted: 25 January 2018

\section{Published online: 20 February 2018}

\section{References}

1. World Health Organization. Noncommunicable diseases country profiles 2014. 2014. http://apps.who.int/iris/bitstream/10665/128038/1/9789241507509_eng. pdf. Accessed 22 Dec 2017

2. Ward BW, Schiller JS, Goodman RA. Multiple chronic conditions among US adults: a 2012 update. Prev Chronic Dis. 2014;11:E62. https://doi.org/10. 5888/pcd11.130389.

3. US Centers for Disease Control and Prevention. Death and Mortality: NCHS FastStats Web Site. 2013. https://www.cdc.gov/nchs/fastats/deaths.htm. Accessed 22 Dec 2017.

4. US Agency for Healthcare Research and Quality. Multiple Chronic Conditions Chartbook: 2010 Medical expenditure panel survey data. AHRQ Publication No Q14-0038. 2014. https://www.ahrq.gov/sites/default/files/ wysiwyg/professionals/prevention-chronic-care/decision/mcc/mccchartbook. pdf. Accessed 22 Dec 2017.

5. Nolte E, Knai C, McKee M. Managing chronic conditions: experience in eight countries. 2008. http://www.euro.who.int/_data/assets/pdf_file/0008/ 98414/E92058.pdf?ua=1. Accessed 22 Dec 2017.

6. Sharma J. Chronic disease managment in the South-East Asia Region: a need to do more. WHO South East Asia J Public Health. 2013;2(2):79-82. https://doi.org/10.4103/2224-3151.122935.

7. Healthcare Information and Management Systems Society. Definitions of mHealth. 2012. http://www.himss.org/definitions-mhealth. Accessed 22 Dec 2017.

8. Bitsaki M, Koutras G, Heep H, Koutras C. Cost-Effective Mobile-Based Healthcare System for Managing Total Joint Arthroplasty Follow-Up. Healthc Inform Res. 2017;23(1):67-73. https://doi.org/10.4258/hir.2017.23.1.67.

9. Hamine S, Gerth-Guyette E, Faulx D, Green BB, Ginsburg AS. Impact of mHealth chronic disease management on treatment adherence and patient outcomes: a systematic review. J Med Internet Res. 2015;17(2):e52. https:// doi.org/10.2196/jmir.3951.

10. Park YT. Emerging New Era of Mobile Health Technologies. Healthc Inform Res. 2016;22(4):253-4. https://doi.org/10.4258/hir.2016.22.4.253.

11. Free C, Phillips G, Felix L, Galli L, Patel V, Edwards P. The effectiveness of Mhealth technologies for improving health and health services: a systematic review protocol. BMC Res Notes. 2010;3:250. https://doi.org/10.1186/17560500-3-250.

12. Fiordelli M, Diviani N, Schulz PJ. Mapping mHealth research: a decade of evolution. J Med Internet Res. 2013;15(5):e95. https://doi.org/10.2196/jmir.2430.

13. Widmer RJ, Collins NM, Collins CS, West CP, Lerman LO, Lerman A. Digital health interventions for the prevention of cardiovascular disease: a systematic review and meta-analysis. Mayo Clin Proc. 2015;90(4):469-80. https://doi.org/10.1016/j.mayocp.2014.12.026.

14. de Ridder M, Kim J, Jing Y, Khadra M, Nanan R. A systematic review on incentive-driven mobile health technology: as used in diabetes management. J Telemed Telecare. 2017;23(1):26-35. https://doi.org/10.1177/1357633X15625539.

15. Cui M, Wu X, Mao J, Wang X, Nie M. T2DM self-management via smartphone applications: a systematic review and meta-analysis. PLoS One. 2016;11(11):e0166718. https://doi.org/10.1371/journal.pone.0166718.

16. Sieverdes JC, Treiber F, Jenkins C. Improving diabetes management with mobile health technology. Am J Med Sci. 2013;345(4):289-95. https://doi org/10.1097/MAJ.0b013e3182896cee.

17. Whitehead $L$, Seaton $P$. The effectiveness of self-management mobile phone and tablet apps in long-term condition management: a systematic review. J Med Internet Res. 2016;18(5):e97. https://doi.org/10.2196/jmir.4883.

18. Liberati A, Altman DG, Tetzlaff J, Mulrow C, Gøtzsche PC, loannidis JP, et al. The PRISMA statement for reporting systematic reviews and meta-analyses of studies that evaluate health care interventions: explanation and elaboration. PLoS Med. 2009;6(7):e1000100. https://doi.org/10.1371/journal.pmed.1000100.

19. Higgins JPT, Altman DG, Sterne JAC. Chapter 8: Assessing risk of bias in included studies. In: Higgins JPT, Green S, editors. Cochrane Handbook for Systematic Reviews of Interventions. 2011. http://handbook-5-1.cochrane. org/chapter_8/8_assessing_risk_of_bias_in_included_studies.htm. Accessed 22 Dec 2017.

20. Bowen DJ, Kreuter M, Spring B, Cofta-Woerpel L, Linnan L, Weiner D, et al. How we design feasibility studies. Am J Prev Med. 2009;36(5):452-7. https:// doi.org/10.1016/j.amepre.2009.02.002.

21. Hebert M. Telehealth success: evaluation framework development. Stud Health Technol Inform. 2001;84(Pt 2):1145-9.
22. Kristjansdottir OB, Fors EA, Eide E, Finset A, Stensrud TL, van Dulmen S, et al. A smartphone-based intervention with diaries and therapist-feedback to reduce catastrophizing and increase functioning in women with chronic widespread pain: randomized controlled trial. J Med Internet Res. 2013;15(1): e5. https://doi.org/10.2196/jmir.2249.

23. Kristjansdottir OB, Fors EA, Eide E, Finset A, Stensrud TL, van Dulmen S, et al. A smartphone-based intervention with diaries and therapist feedback to reduce catastrophizing and increase functioning in women with chronic widespread pain. part 2: 11-month follow-up results of a randomized trial. J Med Internet Res. 2013;15(3):e72. https://doi.org/10.2196/jmir.2442.

24. Dicianno BE, Fairman AD, McCue M, Parmanto B, Yih E, McCoy A, et al. Feasibility of using mobile health to promote self-management in spina bifida. Am J Phys Med Rehabil. 2016;95(6):425-37. https://doi.org/10.1097/ phm.0000000000000400.

25. Ginis P, Nieuwboer A, Dorfman M, Ferrari A, Gazit E, Canning CG, et al. Feasibility and effects of home-based smartphone-delivered automated feedback training for gait in people with Parkinson's disease: a pilot randomized controlled trial. Parkinsonism Relat Disord. 2016;22:28-34. https://doi.org/10.1016/j.parkreldis.2015.11.004.

26. Kearney N, McCann L, Norrie J, Taylor L, Gray P, McGee-Lennon M, et al. Evaluation of a mobile phone-based, advanced symptom management system (ASyMS) in the management of chemotherapy-related toxicity. Support Care Cancer. 2009;17(4):437-44. https://doi.org/10.1007/s00520-008-0515-0.

27. Garcia-Palacios A, Herrero R, Belmonte MA, Castilla D, Guixeres J, Molinari G, et al. Ecological momentary assessment for chronic pain in fibromyalgia using a smartphone: a randomized crossover study. Eur J Pain. 2014;18(6):862-72.

28. Cingi C, Yorgancioglu A, Cingi CC, Oguzulgen K, Muluk NB, Ulusoy S, et al. The "physician on call patient engagement trial" (POPET): measuring the impact of a mobile patient engagement application on health outcomes and quality of life in allergic rhinitis and asthma patients. Int Forum Allergy Rhinol. 2015;5(6):487-97. https://doi.org/10.1002/alr.21468.

29. Hägglund E, Lynga P, Frie F, Ullman B, Persson H, Melin M, et al. Patientcentred home-based management of heart failure. Findings from a randomised clinical trial evaluating a tablet computer for self-care, quality of life and effects on knowledge. Scand Cardiovasc J. 2015;49(4):193-9. https:// doi.org/10.3109/14017431.2015.1035319.

30. Martin SS, Feldman DI, Blumenthal RS, Jones SR, Post WS, McKibben RA, et al. mActive: a randomized clinical trial of an automated mHealth intervention for physical activity promotion. J Am Heart Assoc. 2015;4(11).

31. Piette JD, Striplin D, Marinec N, Chen J, Trivedi RB, Aron DC, et al. A mobile health intervention supporting heart failure patients and their informal caregivers: a randomized comparative effectiveness trial. J Med Internet Res. 2015;17(6):e142. https://doi.org/10.2196/jmir.4550.

32. DeVito Dabbs A, Song MK, Myers BA, Li R, Hawkins RP, Pilewski JM, et al. A randomized controlled trial of a mobile health intervention to promote selfmanagement after lung transplantation. Am J Transplant. 2016;16(7):217280. https://doi.org/10.1111/ajt.13701.

33. Vuorinen AL, Leppanen J, Kaijanranta H, Kulju M, Helio T, van Gils M, et al. Use of home telemonitoring to support multidisciplinary care of heart failure patients in Finland: randomized controlled trial. J Med Internet Res. 2014;16(12):e282. https://doi.org/10.2196/jmir.3651.

34. Cubo E, Mariscal N, Solano B, Becerra V, Armesto D, Calvo S, Arribas J, Seco $J$, Martinez A, Zorrilla L, et al. Prospective study on cost-effectiveness of home-based motor assessment in Parkinson's disease. J Telemed Telecare. 2016;23(2):328-38. https://doi.org/10.1177/1357633X16638971.

35. Lee JA, Evangelista LS, Moore AA, Juth V, Guo Y, Gago-Masague S, et al. Feasibility study of a mobile health intervention for older adults on oral anticoagulation therapy. Gerontol Geriatr Med 2016;2:2333721416672970. doi: https://doi.org/10.1177/2333721416672970.

36. Mera TO, Heldman DA, Espay AJ, Payne M, Giuffrida JP. Feasibility of homebased automated Parkinson's disease motor assessment. J Neurosci Methods. 2012;203(1):152-6. https://doi.org/10.1016/j.jneumeth.2011.09.019.

37. Statista. Number of smartphone users in the United States from 2010 to 2022 (in millions). 2017. https://www.statista.com/statistics/201182/forecastof-smartphone-users-in-the-us. Accessed 22 Dec 2017.

38. Statista. Number of smartphone users worldwide from 2014 to 2020 (in billions). 2017. https://www.statista.com/statistics/330695/number-ofsmartphone-users-worldwide. Accessed 22 Dec 2017.

39. Pew Research Center. Record shares of Americans now own smartphones, have home broadband. 2017. http://www.pewresearch.org/fact-tank/2017/ 01/12/evolution-of-technology. Accessed 22 Dec 2017. 